\title{
Multidisciplinary approach detects speciation within the kissing bug Panstrongylus rufotuberculatus populations (Hemiptera, Heteroptera, Reduviidae)
}

\author{
Sebastián Pita ${ }^{1}{ }^{+}$, Andrés Gómez-Palacio ${ }^{2}$, Pedro Lorite ${ }^{3}$, Jean Pierre Dujardin ${ }^{4}$, \\ Tamara Chavez ${ }^{5}$, Anita G Villacís ${ }^{6}$, Cleber Galvão ${ }^{7}$, Yanina Panzera', Lucía Calleros ${ }^{1}$, \\ Santiago Pereyra-Mello', Gabriela Burgueño-Rodríguez ${ }^{8}$, Francisco Panzera1/+ \\ ${ }^{1}$ Universidad de la República, Facultad de Ciencias, Sección Genética Evolutiva, Montevideo, Uruguay \\ ${ }^{2}$ Universidad Pedagógica y Tecnológica de Colombia, Laboratorio de Investigación en Genética Evolutiva, Boyacá, Colombia \\ 3Universidad de Jaén, Departamento de Biología Experimental, Área de Genética, Jaén, Spain \\ ${ }^{4}$ University of Montpellier, Institut de Recherche pour le Développement, International Campus in Baillarguet, Montpellier, Occitanie, France \\ ${ }^{5}$ Instituto Nacional de Laboratorios de Salud, Laboratorio de Entomología Médica, La Paz, Bolivia \\ ${ }^{6}$ Pontificia Universidad Católica del Ecuador, Facultad de Ciencias Exactas y Naturales, Escuela de Ciencias Biológicas, Centro de Investigación \\ para la Salud en América Latina, Nayón, Quito, Ecuador \\ ${ }^{7}$ Fundação Oswaldo Cruz-Fiocruz, Instituto Oswaldo Cruz, Laboratório Nacional e Internacional de Referência em Taxonomia \\ de Triatomíneos, Rio de Janeiro, RJ, Brasil \\ ${ }^{8}$ Universidad de la República, Centro Universitario Regional, Departamento de Ciencias Biológicas, Laboratorio de Genética \\ Molecular Humana, Salto, Uruguay
}

BACKGROUND Panstrongylus rufotuberculatus (Hemiptera-Reduviidae) is a triatomine species with a wide geographic distribution and a broad phenotypic variability. In some countries, this species is found infesting and colonising domiciliary ecotopes representing an epidemiological risk factor as a vector of Trypanosoma cruzi, etiological agent of Chagas disease. In spite of this, little is known about $P$. rufotuberculatus genetic diversity.

METHODS Cytogenetic studies and DNA sequence analyses of one nuclear (ITS-2) and two mitochondrial DNA sequences (cyt $b$ and $c o I$ ) were carried out in P. rufotuberculatus individuals collected in Bolivia, Colombia, Ecuador and Mexico. Moreover, a geometric morphometrics study was applied to Bolivian, Colombian, Ecuadorian and French Guiana samples.

OBJECTIVES To explore the genetic and phenetic diversity of $P$. rufotuberculatus from different countries, combining chromosomal studies, DNA sequence analyses and geometric morphometric comparisons.

FINDINGS We found two chromosomal groups differentiated by the number of $\mathrm{X}$ chromosomes and the chromosomal position of the ribosomal DNA clusters. In concordance, two main morphometric profiles were detected, clearly separating the Bolivian sample from the other ones. Phylogenetic DNA analyses showed that both chromosomal groups were closely related to each other and clearly separated from the remaining Panstrongylus species. High nucleotide divergence of $c y t b$ and coI fragments were observed among P. rufotuberculatus samples from Bolivia, Colombia, Ecuador and Mexico (Kimura 2-parameter distances higher than 9\%).

MAIN CONCLUSIONS Chromosomal and molecular analyses supported that the two chromosomal groups could represent different closely related species. We propose that Bolivian individuals constitute a new Panstrongylus species, being necessary a detailed morphological study for its formal description. The clear morphometric discrimination based on the wing venation pattern suggests such morphological description might be conclusive.

Key words: Chagas disease vectors - cytochrome b gene - cytochrome C oxidase subunit I - internal transcribed spacer 2 - karyotype evolution - morphometric analyses - sex chromosome fusion

The Triatominae subfamily (Hemiptera: Heteroptera: Reduviidae) includes more than 150 blood-sucking species grouped into 16 genera $^{(1,2)}$ These insects act as vectors of Trypanosoma cruzi, the etiological agent of Chagas disease, which is recognised as the most seri-

\section{doi: 10.1590/0074-02760210259}

Financial support: From Uruguay: MEC/DICYT/II/FVF/2019/054 and CSICUdelar (project grant No. 160); From Spain: Spanish Junta de Andalucía by AUIP and "Programa Operativo FEDER Andalucía 2014-2020". UNICEF/ UNPD/World Bank/WHO Special Programme for TDR [A20785], PAHO [A60655], and Pontificial Catholic University of Ecuador (L13254).

+ Corresponding authors: spita@fcien.edu.uy/fcopanzera@gmail.com

(1) https://orcid.org/ 0000-0002-4102-5808

(1) https://orcid.org/ 0000-0001-5148-957X

Received 02 August 2021

Accepted 16 November 2021 ous human parasitic disease of Latin America, affecting 5-6 million people. ${ }^{(2,3)}$ In the absence of vaccines or adequate drugs for large-scale treatment, the reduction of disease incidence depends mainly on vector control of bug's populations in human dwellings. ${ }^{(2,3)} \mathrm{An}$ accurate taxonomic identification and knowledge about the genetics of these insects are keys to ensure successful entomological surveillance after control campaigns. ${ }^{(2)}$

The Panstrongylus genus belongs to the Triatomini tribe and is included within the North American lineage, constituting a paraphyletic group. ${ }^{(4,5,6)}$ Panstrongylus is the third most relevant genus within Triatominae in terms of species richness (one fossil and 14 living species) and epidemiological impact. ${ }^{(7)}$ Among these species, Panstrongylus megistus is the most significant Chagas disease vector, particularly in Brazil. Although 
the remaining Panstrongylus species are primarily sylvatic, some of them have gained attention, as they are involved in domiciliation processes and hence the transmission of Chagas disease to humans. Panstrongylus rufotuberculatus is one of these species, with a wide distribution area extending from Mexico to Argentina, from lowland rainforests to arid highlands of up to $2600 \mathrm{~m}$ above sea level. ${ }^{(1,7)}$ Additionally it is adapted to dry as well as humid ecotopes, being found in a great variety of sylvatic hosts including armadillos, kinkajous, opossums, rodents, bats and birds burrows. ${ }^{(1)}$ Furthermore, several authors reported breeding colonies of P. rufotuberculatus inside and around dwellings in Bolivia, ${ }^{(8,9,10)}$ Colombia, ${ }^{(11)}$ Ecuador, ${ }^{(12,13)}$ Peru $^{(14)}$ and Venezuela. ${ }^{(15)}$ Some studies highlight the high rates of house infestation $^{(10,11)}$ and infection with Trypanosoma cruzi. ${ }^{(12)}$ The domiciliary presence of $P$. rufotuberculatus represents an important epidemiological risk factor for Chagas disease transmission in several Latin American countries, mainly in areas where the principal vector (e.g. Triatoma infestans) is absent due to vector control programs. ${ }^{(10)}$

Following the description of the male holotype from Panama by Champion (1899), several authors have emphasised the chromatic, morphologic and morphometric variation of $P$. rufotuberculatus along their geographical distribution. ${ }^{(8,9,16)}$ For example, the carinae limiting the central depression of the scutellum is entirely black in specimens from Bolivia, Brazil, Costa Rica, Peru and Venezuela, while it is red or reddish in Colombia, Panama and Suriname insects. ${ }^{(8,16)}$ A traditional morphometric analysis of head capsules of individuals found in the domiciles from Bolivia (La Paz) versus sylvatic specimens deposited in the collection of the Natural History Museum of London (NHM), from Mexico, Ecuador and Panama (including the holotype of $P$. rufotuberculatus), showed a consistent variation of metric properties. ${ }^{(9)}$ Until now, no geometric morphometric approach had been applied.

As other hemipteran species, Panstrongylus genus presents holocentric chromosomes characterised by the absence of a primary constriction. Chromosomal analyses of eight out of the 14 Panstrongylus species reveal that this genus is variable in its diploid chromosome number (21, 23 or 24 chromosomes in males), including the number of autosomes (18 and 20), sex chromosome systems $\left(\mathrm{X}_{1} \mathrm{X}_{2} \mathrm{Y}\right.$ and $\left.\mathrm{X}_{1} \mathrm{X}_{2} \mathrm{X}_{3} \mathrm{Y}\right)$ and different amount of autosomal $\mathrm{C}$-heterochromatin. ${ }^{(17)}$ Previous cytogenetic studies on P. rufotuberculatus were restricted to individuals from Colombia (Antioquia and Santander) showing a male diploid chromosome number of 23 chromosomes (20 autosomes plus $\mathrm{X}_{1} \mathrm{X}_{2} \mathrm{Y}$ ) and C-heterochromatic regions on most autosomes. ${ }^{(18)}$

We examined the cytogenetic characteristics of $P$. rufotuberculatus individuals collected in several localities from three countries (Bolivia, Colombia and Ecuador). This material was also employed to address wing morphometrics analyses, which included an additional sample from French Guiana not analysed by cytogenetics. We determined, for the first time, the chromosomal position of major ribosomal loci (rDNA clusters) by fluorescence in situ hybridisation (FISH). This chromosomal trait is considered as species specific and very useful to distinguish chromosomally undifferentiated species, such as observed in several species complexes..$^{(19,20,21)}$ In order to check the phylogenetic relationships of these individuals, sequences analyses of one nuclear (internal transcribed spacer 2 - ITS-2) and two mitochondrial DNA fragments (cytochrome b - cyt $b$ - and cytochrome C oxidase subunit I - coI) were performed, including sequences of $P$. rufotuberculatus from different countries and others Panstrongylus spp. available in GenBank.

\section{MATERIALS AND METHODS}

Materials - The specimens were identified according to morphological keys established in Lent and Wygodzinsky.(1) Table I and Fig. 1 show the geographic origin and number of $P$. rufotuberculatus individuals studied by morphometric, cytogenetics and molecular analyses (including its GenBank accession numbers). Previously published cytogenetic data are also incorporated. ${ }^{(18)}$

In our phylogenetic analyses, we used all Panstrongylus sequences available in GenBank, in addition to sequences obtained by us. However, several ITS-2 and cyt $b$ sequences identified as $P$. chinai, $P$. howardi and P. rufotuberculatus, submitted in GenBank in 2014 by Sempertegui-Sosa et al., were not included (Unpublished data). Barnabé et al. ${ }^{(13)}$ recognised, in several of these sequences, labeling errors in their species identification. Since Panstrongylus species belong to the North American Triatomini lineage, other species from this lineage were also included, as well as several species from the South American lineage as out-groups. Since there were few $c o I$ sequences available in GenBank for the fragment employed in the present paper, we have sequenced individuals of other Panstrongylus species: P. chinai (MZ643675); P. lignarius/herreri (MZ643676); P. geniculatus (MZ643678, MZ643679) and P. tupynambai (MZ643677).

Morphometric study - The wings pictures assembled for these morphometric studies were provided by different authors (TC, AV, JPD and two others cited in the acknowledgments). Since the pictures from Bolivia and Colombia did not harbor a size scale, our statistical analyses of the total sample were restricted to shape comparisons only, without including size analyses.

Six landmarks were selected on the wings in addition to 14 semilandmarks that were used to capture the curved lines of veins between landmarks (Fig. 2). All landmarks and semilandmarks were submitted to partial Procrustes superimposition (GPA) ${ }^{(22)}$ and semilandmarks then subjected to sliding procedure. ${ }^{(23)}$ The tangent space projections of 67 individuals were used as input for a principal component analysis (PCA). The discriminant analysis used the nine first PCA as input, and was illustrated by the factor map of first and second discriminant factor (Fig. 3). This analysis compared males, females and countries as separated groups. Because of the very small samples from Colombia, this country was mixed with the neighbor country Ecuador.

Cytogenetic studies - We analysed 33 P. rufotuberculatus bugs whose geographical origin is detailed in Table I. Gonads were removed from adult insects and fixed in ethanol-acetic acid (3:1). C-banding and fluo- 


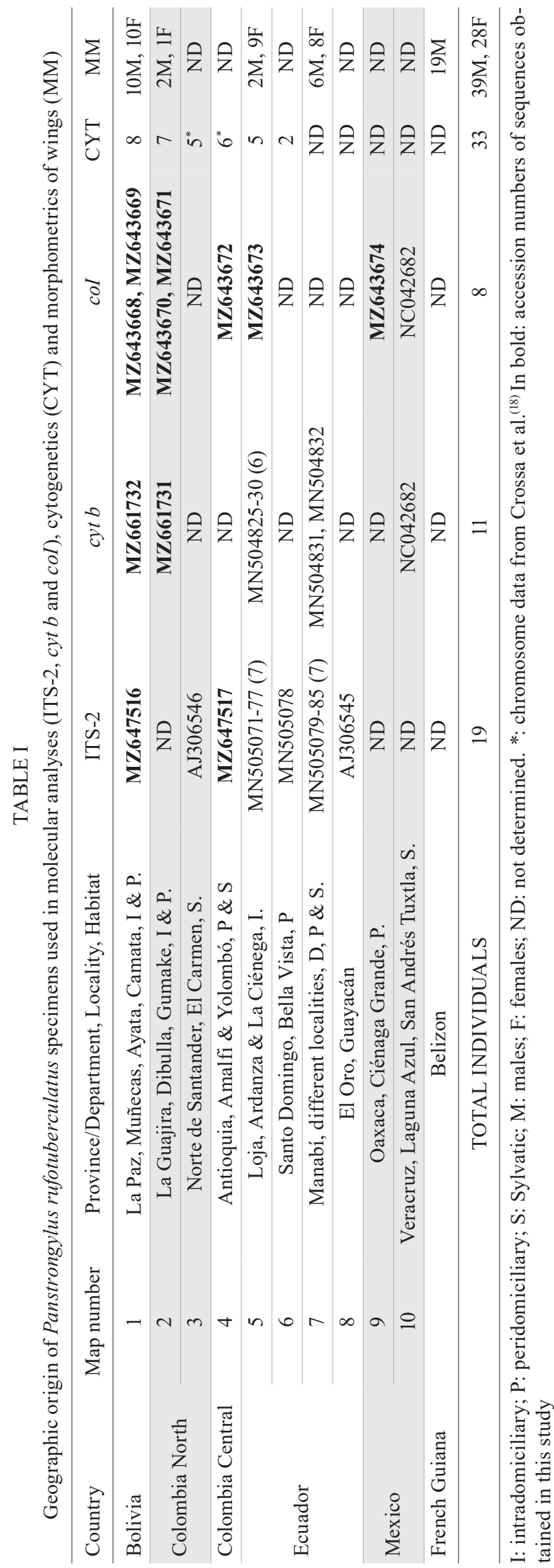

rescent in situ hybridisation (FISH) using as a probe an 18S rDNA fragment (included within 45S rDNA clusters) were carried out on chromosome preparations obtained by the squash method. ${ }^{(18,19)}$ Slides were examined under a Nikon Eclipse 80i microscope and the images were obtained with a DS-5Mc-U2 digital camera. For each specimen, at least 20 cells were analysed to determine the chromosome number, the $\mathrm{C}$-banding pattern and the chromosomal location of the 45S rDNA clusters. The C-banding pattern of $P$. rufotuberculatus was previously described on individuals from Colombia (Antioquia and Santander). ${ }^{(18)}$ In this paper, we included more individuals from the previously reported populations, as well as from other localities in Colombia (La Guajira and Yolombó), Ecuador (Loja and Santo Domingo) and Bolivia (La Paz). The chromosomal locations of $45 \mathrm{~S}$ ribosomal clusters were determined by FISH in P. rufotuberculatus individuals and described here for the first time. To elucidate the possible origin of the sex chromosome polymorphism, we estimate the relative length (in percent) of the X chromosome(s) in relation to the chromosome complement total length. Images were analysed using the Nikon Nis Elements 3.1. Advanced Software. We analysed 100 metaphase I and II images from three individuals from each sex chromosome system.

DNA extraction and sequencing - Bugs legs, stored in $70 \%$ ethanol, were used for DNA extraction using standard phenol-chloroform technique. A $210 \mathrm{bp} \mathrm{ITS-2}$ fragment was amplified by PCR using the 5.8T and 28T primers ${ }^{(4)}$ a $600 \mathrm{bp}$ fragment of $c o I$ was amplified with ACO and COIb primers, ${ }^{(24)}$ and a 419 bp fragment of cyt $b$ with the primers CYTB7432F and CYTB7433R. (25) PCR products were Sanger-sequenced by Macrogen Inc. (Korea). Forward and reverse sequences were aligned using Bioedit v.7.0.9.

Genetic diversity and phylogenetic analyses - Considering that ITS-2 nuclear sequences have wide size differences among species, gaps (indels) were included as informative characters in sequence-based and alignment analyses ${ }^{(26)}$ and added as an additional block into the data file using FastGap v.1.2 software (http://www. aubot.dk/FastGap_home.htm).

For the three molecular markers, MAFFT v7.453 was employed to align sequences. Genetic distances for mitochondrial markers were calculated using APE package v5.4.1 ${ }^{(27)}$ in $\mathrm{R}$ v3.6.1, using the Kimura 2-parameter substitution model (K2-p). The best fitting substitution models for the mitochondrial genes were estimated using Smart Model Selection and jModelTest v.2.1.10 ${ }^{(28)}$ for the nuclear ITS-2. Decisions were taken under Bayesian information criteria (BIC).

Maximum likelihood (ML) phylogenetic trees were obtained using PhyML v3. ${ }^{(29)}$ Bootstrap values were estimated from 1000 replicates. In order to include indels information for ITS-2 sequences, a Bayesian phylogenetic tree was inferred with MrBayes v.3.2.7a. ${ }^{(30)}$ The posterior probability of the phylogenetic tree was estimated by Metropolis-coupled Markov chain Monte Carlo (MCMCMC). The MCMCMC settings consisted of two simultaneous, independent runs with four chains each 


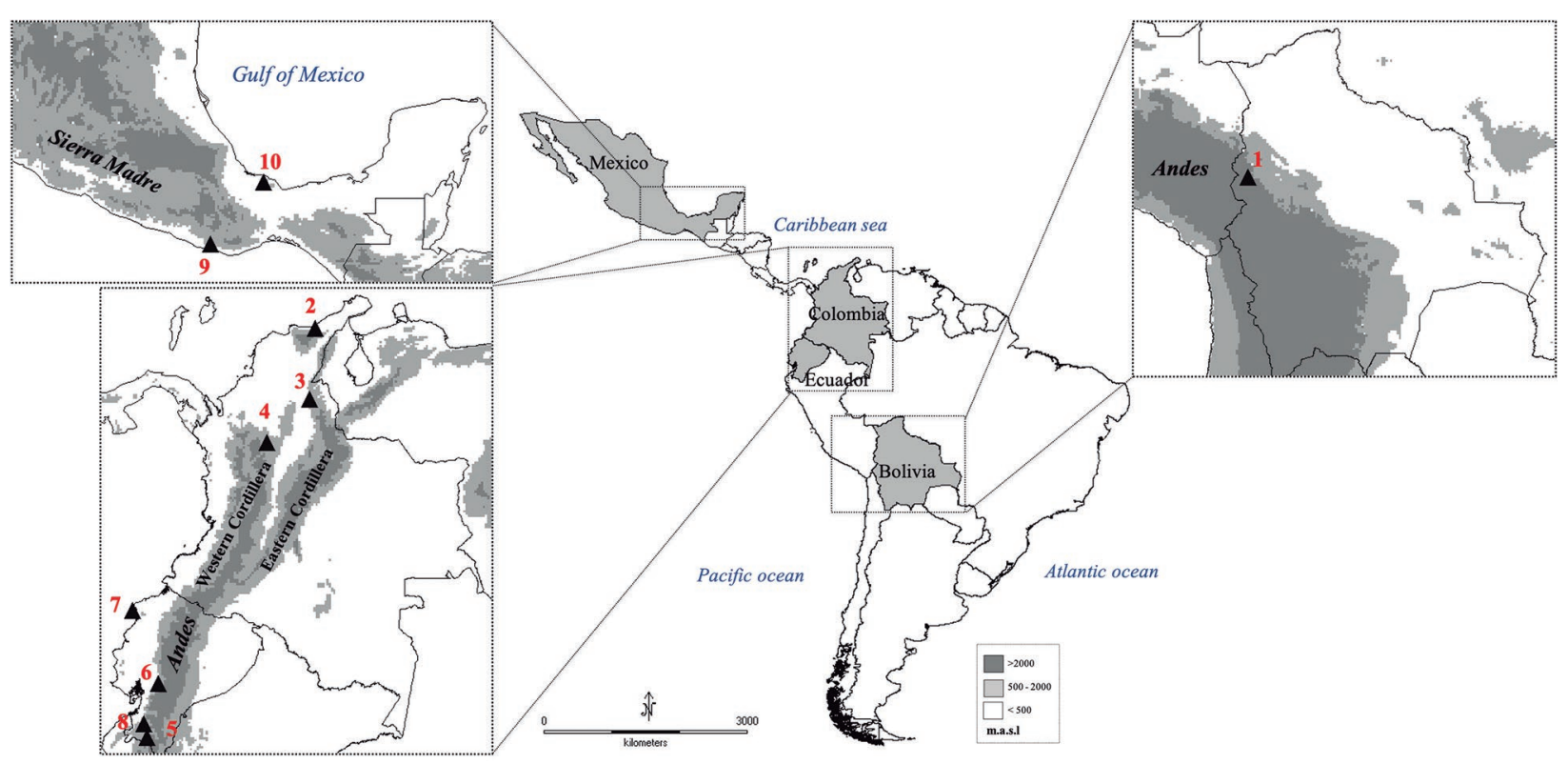

Fig. 1: Latin American map showing the geographic location of the 10 sampling sites of Panstrongylus rufotuberculatus individuals studied by genetic markers in this paper. Site names and other details are given in Table I.

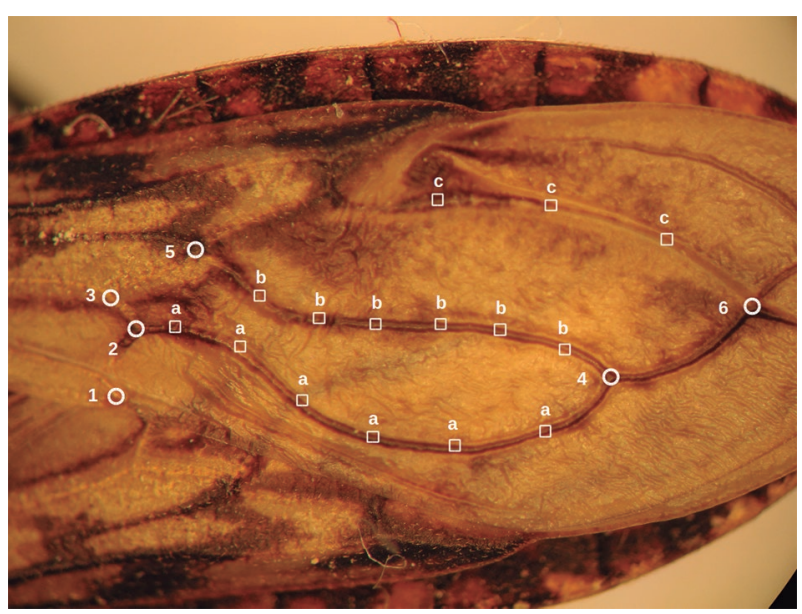

Fig. 2: landmarks and semilandmarks of the wing venation in Panstrongylus rufotuberculatus. Numbers correspond to true landmarks type I (LM I) and letters to semilandmarks. The semilandmarks are partly capturing the shape of three veins: the anal vein (group "a"), the cubital vein (group "b") and the median vein (group "c"). Their positions were computed depending on the position of two flanking landmarks.

(with default heating temperature 0.1), which were run for $10 \times 6$ generations and sampled every 1000 generations with a $25 \%$ burn-in. Convergence was assessed using the average standard deviation of split frequency values $(<$ 0.01 ) and by the Effective Sample Size (ESS > 200).

\section{RESULTS}

Morphometric variation (Fig. 3) - On the discriminant space derived from shape variables, males and females were grouped together as expected. The first discriminant factor (DF) represented $77 \%$ of the total shape variation among samples (Fig. 3). On this first axis,

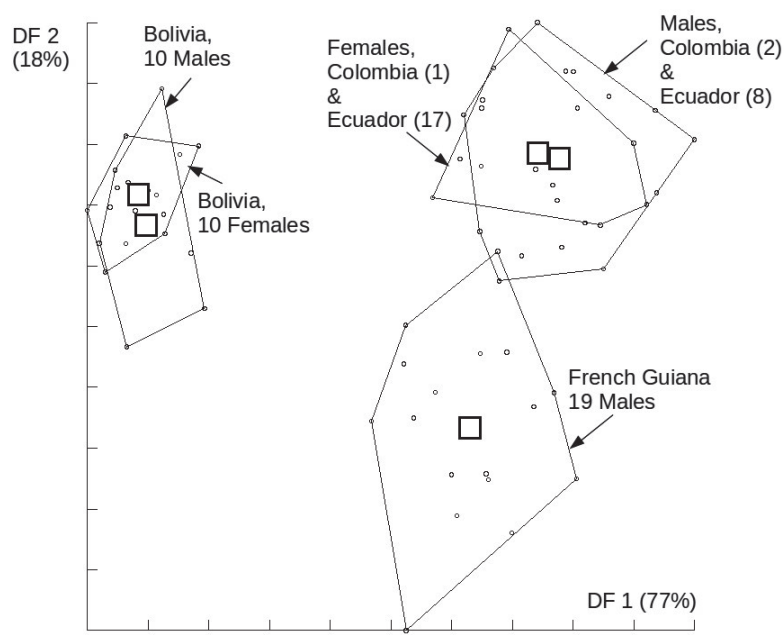

Fig. 3: factor map of the two first discriminant factors (DF 1, horizontal axis and DF 2, vertical axis) derived from first nine principal components which together represented $73 \%$ of the total shape variation in Panstrongylus rufotuberculatus. The contribution of each DF to the total discriminant space is indicated between brackets. Bolivia is completely distinguished on DF1, French Guiana is distinguished on DF2.

males and females from Bolivia constituted a completely separated group. The second DF represented $18 \%$ of the total shape variation, separating the Guiana sample from the others, but not completely from the Colombia-Ecuador sample. The remaining 2 DF represented only $4 \%$ of the total variation and were not presented here.

C-banding and fluorescence in situ hybridisation (Fig. 4) - Chromosomal analyses of 33 P. rufotuberculatus individuals from Bolivia, Colombia and Ecuador identified two chromosomal groups, also called patterns or cytotypes, with differences in the number of sex 

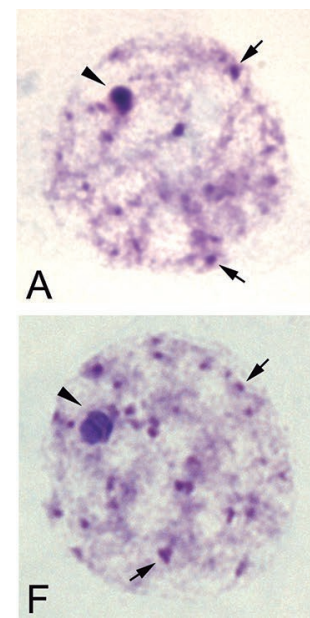
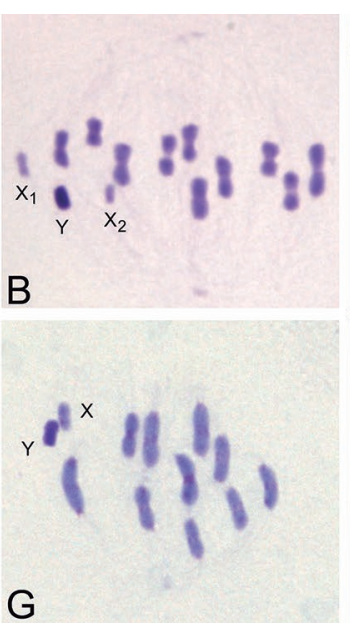
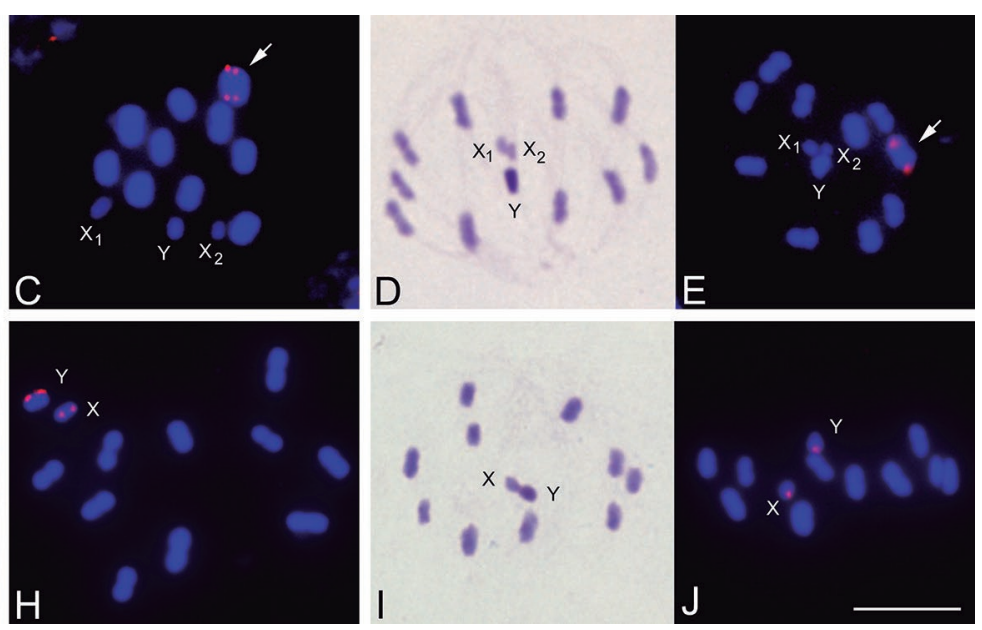

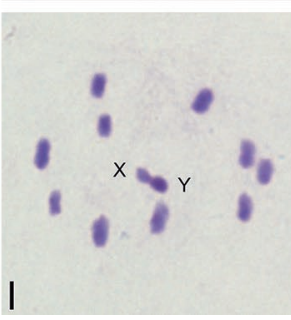

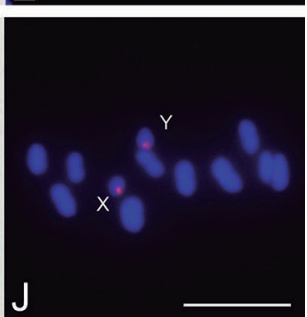

Fig. 4: male meiosis in Panstrongylus rufotuberculatus. Top row (A-E): P. rufotuberculatus from Colombia and Ecuador named cytotype 1 (2n $=23=20 \mathrm{~A}+\mathrm{X}_{1} \mathrm{X}_{2} \mathrm{Y}$ ) showing two $\mathrm{X}$ chromosomes and $45 \mathrm{~S}$ ribosomal clusters on one autosome. Bottom row (F-J): P. rufotuberculatus from Bolivia named cytotype $2(2 n=22=20 \mathrm{~A}+\mathrm{XY})$ showing only one $\mathrm{X}$ chromosome and ribosomal clusters in $\mathrm{X}$ and $\mathrm{Y}$ chromosomes. C-banding: A, B, D, F, G and I. Fluorescent in situ hybridization with $18 \mathrm{~S}$ ribosomal DNA probe: C, E, H and J. Chromosomes in blue (DAPI stain) and ribosomal signals (45S rDNA clusters) in red on one autosome (arrows in $\mathrm{C}$ and $\mathrm{E}$ ) or on the $\mathrm{X}$ and $\mathrm{Y}$ chromosomes (H and $\mathrm{J}$ ). (A and $\mathrm{G})$ : First meiotic prophases showing $\mathrm{C}$-dots dispersed in the nuclei. First and second meiotic metaphases (B, C, G, H and D, E, I, J respectively). Bar $=10 \mu \mathrm{m}$.

chromosomes and in the chromosomal location of $45 \mathrm{~S}$ ribosomal clusters. Both cytotypes presented autosomal C-heterochromatin located at one or both chromosomal ends in almost all autosomal pairs.

Cytotype 1: Individuals from Colombian and Ecuadorian populations (Fig. 4A-E, top row,). Male individuals presented a diploid number (2n) of 23 chromosomes, with 10 autosomal pairs plus three sex chromosomes: $\mathrm{X}_{1} \mathrm{X}_{2} \mathrm{Y}$. Females have $2 \mathrm{n}=24$ (20 autosomes plus $\mathrm{X}_{1} \mathrm{X}_{2} \mathrm{X}_{1} \mathrm{X}_{2}$ ). Early meiotic prophase was characterised by the presence of a largest heterochromatic chromocenter constituted by the association of the sex chromosomes (Fig. 4A, arrowhead). Furthermore, several heterochromatic dots were scattered throughout the nucleus (Fig. 4A, arrows), corresponding with terminal C-regions of autosomal pairs. Autosomal chromosomes showed scarce variation regarding their size (Fig. 4BE). The Y sex chromosome was middle sized and totally C-heterochromatic, while $\mathrm{X}_{1}$ and $\mathrm{X}_{2}$ chromosomes were the smallest of the complement and had an intermediate staining (Fig. 4B,D). In metaphase II, the three sex chromosomes disposed as a pseudotrivalent, in the center of an autosomal ring-like shape. FISH results indicated that 45S rDNA clusters were located on the largest autosomal pair (Fig. 4C, E, arrows).

Cytotype 2: Individuals from Bolivian population (Fig. 4F-J, bottom row). All individuals presented a diploid number (2n) of 22 chromosomes, with 10 autosomal pairs plus two sex chromosomes (XY in males and XX in females). Autosomal C-heterochromatin distribution was similar to what was observed in individuals from Colombia and Ecuador (Fig. 4A, F). In addition, the Y sex chromosome was middle sized and totally heterochromatic. The $\mathrm{X}$ chromosome was similar in size to the Y chromosome (Fig. 4G-I) with an intermediate staining. During metaphase II, the $\mathrm{X}$ and $\mathrm{Y}$ chromosomes formed a pseudobivalent (Fig. 4I). The 45S rDNA clusters were situated on both sex chromosomes (X and Y) (Fig. 4H, J).
To clarify the origin of the sex chromosome polymorphism, we measured the average length of the $\mathrm{X}$ chromosomes over the total length of the chromosome complement. In Bolivian male individuals, presenting $\mathrm{XY}$ sex chromosomes, the mean length of the $\mathrm{X}$ chromosome represented $5.98 \pm 0.17 \%$. In the individuals from Colombian and Ecuadorian populations, the sum of the $\mathrm{X}_{1}$ and $\mathrm{X}_{2}$ chromosomes average lengths represented $5.87 \pm 0.15 \%$.

Nuclear ITS-2 (Fig. 5) - The best fitted model was the HYK $+\mathrm{G}$ and selected for the construction of the ITS-2 Bayesian tree. This phylogenetic tree included sequences of the five Panstrongylus species together with 19 sequences of $P$. rufotuberculatus available in GenBank, two of them sequenced in this study (Table I). Identical sequences were grouped in the same haplotype, so only one was represented in the tree [Supplementary data (Table)]. Since Panstrongylus species belong to the North American lineage, other triatomine species from this lineage were also included. South American lineage species are considered as out-groups. This analysis showed that the Panstrongylus genus is clearly paraphyletic. All P. rufotuberculatus sequences (four haplotypes representing 19 sequences) are grouped together in one clade with high statistical support, and also separated from the remaining five Panstrongylus species.

Mitochondrial cyt b fragment (Fig. 6) - Maximum likelihood (HKY85+G+I) analysis using $c y t b$ fragment sequences of seven Panstrongylus species plus P. rufotuberculatus is shown in Fig. 6 (collapsed tree). This phylogenetic analysis depicted the genus Panstrongylus as a paraphyletic clade. The 11 sequences identified as $P$. rufotuberculatus were grouped into two well supported clades. One of them includes the sequences from Bolivia and Colombia (La Guajira) and the other one the remaining sequences from Ecuador and Mexico. Uncollapsed tree could be seen in Supplementary data (Figure). 

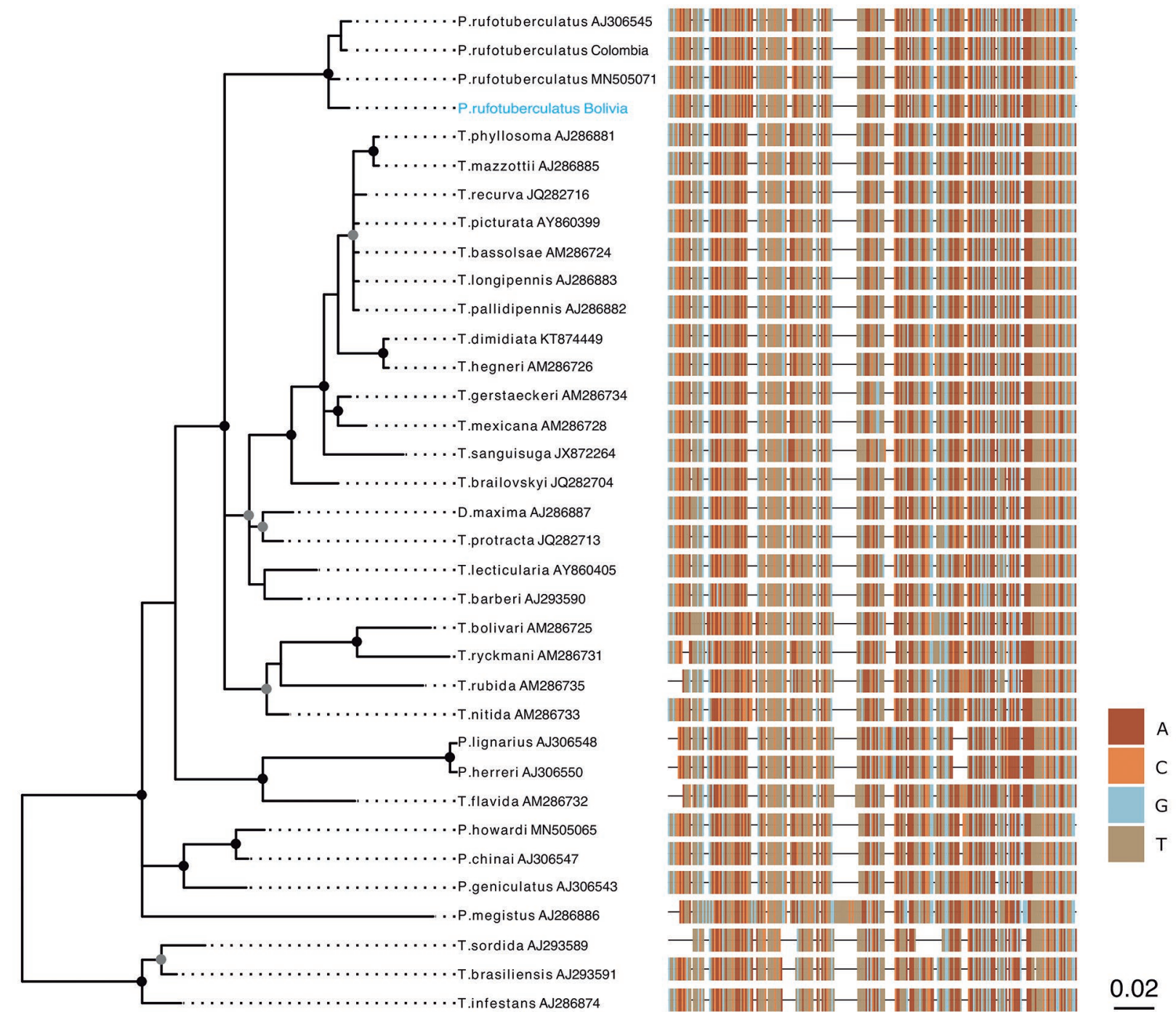

Fig. 5: Bayesian phylogenetic tree obtained from ITS-2 fragments including the sequences of the six Panstrongylus species available in GenBank (P. lignarius and P. herreri counted as one species). The Panstrongylus genus is paraphyletic; with $P$. rufotuberculatus clade clearly separated from the other Panstrongylus species. The 19 individuals of $P$. rufotuberculatus, showed as four haplotypes, are clustered in one clade, including P. rufotuberculatus from Bolivia. Circles represent statistical support obtained through posterior probability, gray circles denote above 0.75 and black circles above 0.9. DNA sequence alignment is represented at the right panel, where each color represents a different DNA base. Accession numbers of P. rufotuberculatus from Bolivia and Colombia are MZ647516 and MZ647517 respectively.

Mitochondrial col fragment (Fig. 7) - The GTR+G+I substitution model was selected for the construction of the coI ML tree. This analysis included five Panstrongylus species plus sequences of $P$. rufotuberculatus. This genetic marker also supports the paraphyly of Panstrongylus. All individuals (eight) initially identified as $P$. rufotuberculatus are closely related to each other constituting a clearly separated clade from the remaining Panstrongylus species. Within this clade, it is possible to identify four well supported subclades: (a) Mexican individuals from Oaxaca and Veracruz, (b) Ecuadorian and Central Colombia (Antioquia) individuals, (c) North Colombia (La Guajira), and (d) Bolivian specimens. The specimens from Bolivia appear as a sister group of $P$. rufotuberculatus from Colombia (La Guajira).
Average genetic distances (K2-p) among the four $P$. rufotuberculatus groups vary between $10.0 \%$ to $19.7 \%$ for $c y t b$ and from $9.0 \%$ to $15.8 \%$ for $c o I$ fragments. Comparing P. rufotuberculatus from Bolivia with the other groups, the average genetic distances are close to the highest values, varying between $10.7 \%$ and $18.7 \%$ for cyt $b$ and $10.6 \%$ and $15.8 \%$ for $c o I$ fragments. For both markers, the closest group to Bolivian individuals is the one from North Colombia (La Guajira): K2-p of 10.7\% for $c y t b$ and $10.6 \%$ for $c o I$ (Table II).

\section{DISCUSSION}

Wing venation patterns - Due to the incomplete information about the wings size, no comparisons of size were possible, and its possible effect on the shape variables as extracted from our landmarks and semiland- 
marks remains unknown. Our work hypothesis considers that the possible allometric effect on shape variables did not bias the global comparison.

Shape as disclosed by the geometric morphometrics approach is a polygenic character ${ }^{(31)}$ and most of its variation has been attributed to genetic drift rather than to environmental conditions. ${ }^{(32)}$ For this reason, the shapebased discriminant space is frequently reminiscent of the geographic map, as in our case here (Fig. 3).

The clear cut shape-based discrimination obtained between the Bolivian sample and the remaining ones parallels the separate position of the Bolivian origin in the morphospace of the head capsule. ${ }^{(9)}$ Considering the wide geographic sample of $P$. rufotuberculatus individuals, the observed morphometric variation pattern is likely to be the reflection of genetic drift. However, the morphometric approach by itself cannot tell us whether it is due to a spatial or biological isolation.

Sex chromosome polymorphism in Heteroptera - In Triatominae as well as in Reduviidae, it is generally agreed that $\mathrm{XX} / \mathrm{XY}$ is the ancestral sex chromosome system. Sex mechanisms with multiple $\mathrm{X}$ or $\mathrm{Y}$ chromosomes are believed to be most probably origi-

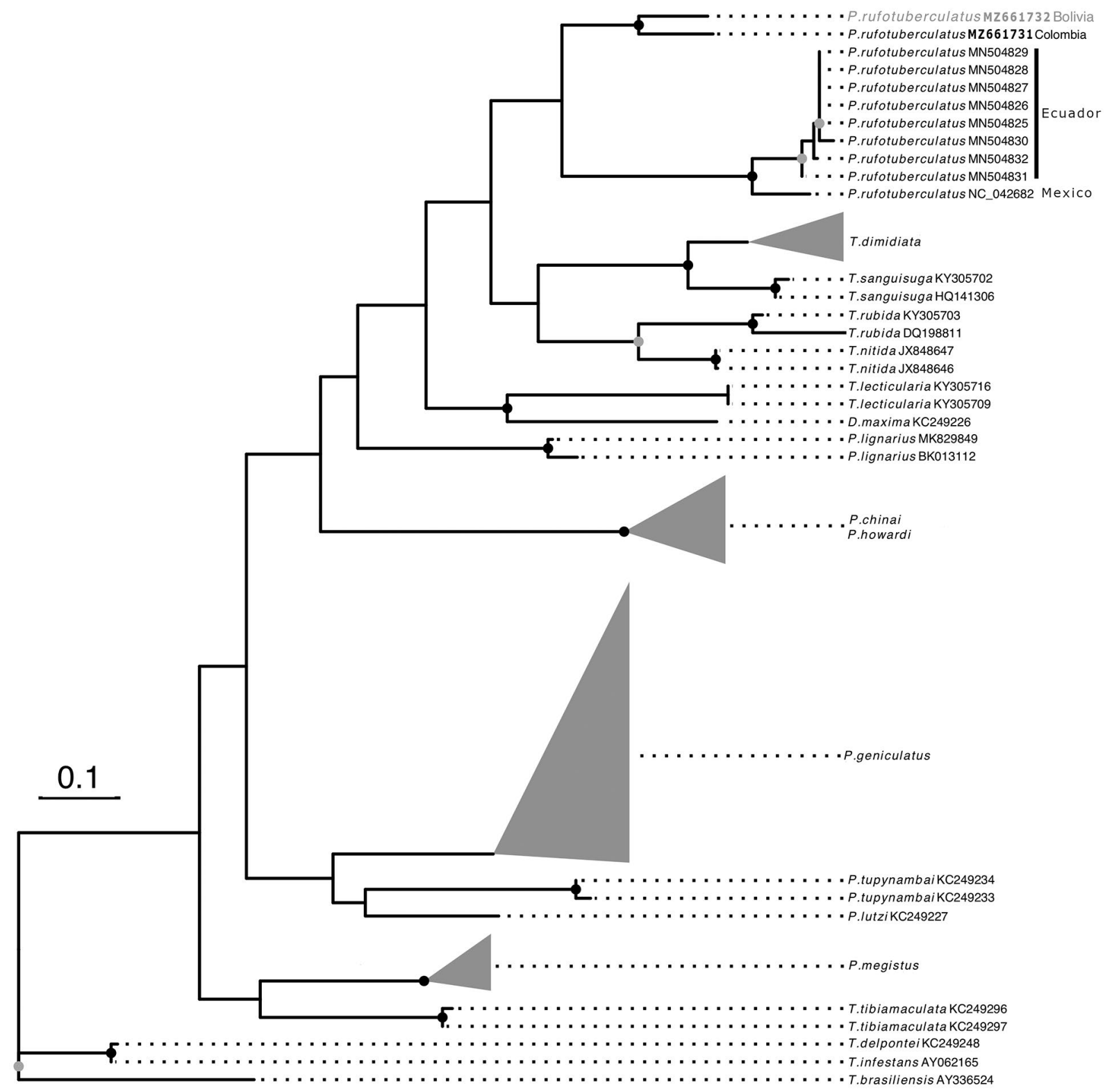

Fig. 6: collapsed maximum likelihood phylogenetic tree obtained from cytochrome b ( cyt b) fragment, including all sequences of Panstrongylus species available in GenBank. The paraphyly of the Panstrongylus genus is clearly shown. The 11 individuals recognized as $P$. rufotuberculatus were grouped into two well supported clades, clearly separated from the remaining seven Panstrongylus species. The individual from Bolivia (in blue) is closely related to $P$. rufotuberculatus from Colombia (La Guajira). Accession numbers of sequences presented herein are depicted in bold. Gray circles denote bootstraps support above 0.75 and black circles above 0.9. Uncollapsed tree could be seen in Supplementary data (Figure). 


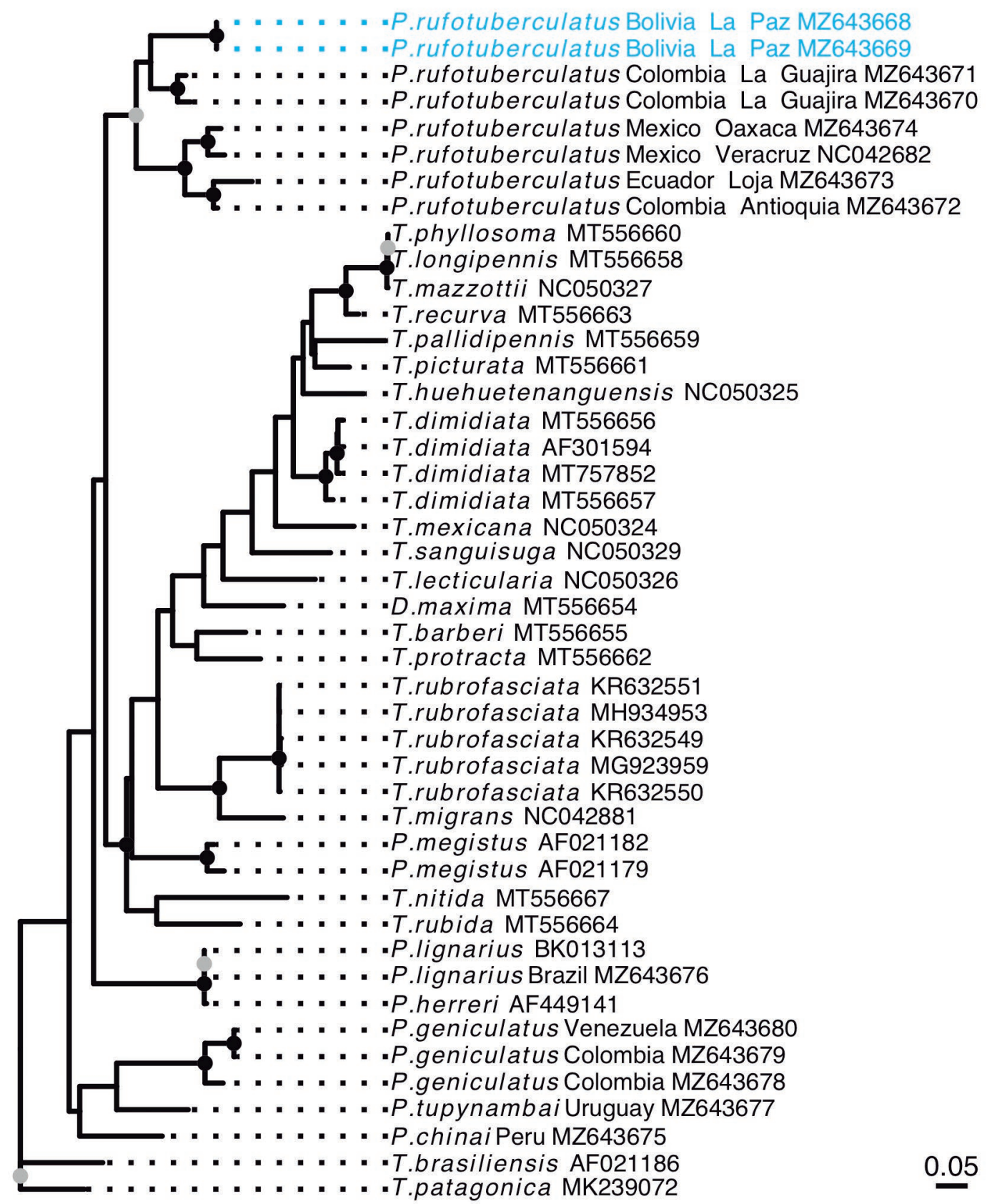

Fig. 7: maximum likelihood phylogenetic tree obtained from cytochrome C oxidase subunit I (coI) fragment from six Panstrongylus species, including all other triatomine species available in GenBank. The genus Panstrongylus is clearly paraphyletic. The eight individuals of $P$. rufotuberculatus are grouped in the same clade, clearly separated from the remaining five Panstrongylus species. Depicted in blue are $P$. rufotuberculatus from Bolivia. Gray circles denote bootstraps support above 0.75 and black circles above 0.9 .

nated through fragmentation(s) of the ancestral $\mathrm{X}$ and $\mathrm{Y}$ chromosome, respectively. ${ }^{(33)}$ Intraspecific variation in the number of sex chromosomes (consider as polymorphisms) are very uncommon. It has been detected in only ten of the more than 1,000 heteropteran species analysed so far. ${ }^{(33,34,35)}$ Almost all of these variations were due to the fragmentation of the $\mathrm{X}$ chromosome, which was supported both by their meiotic chromosomal behavior and by the size comparison of the sex chromosomes between the individuals with simple system (XY) and those with multiple systems $(\mathrm{XnY}) \cdot{ }^{(34,35)} \mathrm{Up}$ to now, intraspecific variation in the number of sex chromosomes has not been reported in the Triatominae subfamily.

Almost all species from the North American Triatomini lineage (38 out of 41), which includes all Panstrongylus species, presented multiple $\mathrm{X}$ chromosomes. According to Panzera et al., ${ }^{(17)}$ the $\mathrm{X}_{1} \mathrm{X}_{2} \mathrm{Y}$ chromosome sex system is considered as the ancestral one in this lineage. Therefore, the XY mechanism observed in Bolivian individuals could represent a derived character originated 
TABLE II

Mitochondrial DNA sequence differentiation among four Panstrongylus rufotuberculatus geographic groups represented in Table I. Mean Kimura 2-parameter (K2-p) distances (in percentages) computed with cyt b (above diagonal) and $c o I$ (below diagonal) partial sequences; mean within-group distances ( $c y t b / c o I)$ are on the diagonal

\begin{tabular}{lcccc}
\hline Cyt b / coI & Mexico & Ecuador + Central Colombia & North Colombia (Guajira) & Bolivia (La Paz) \\
\hline Mexico & $0 / 3.93$ & 10.04 & 19.72 & 18.75 \\
\hline Ecuador + Central Colombia & 9.00 & $1.56 / 5.42$ & 16.65 & 18.06 \\
\hline North Colombia (Guajira) & 12.78 & 12.30 & $0 / 2.64$ & 10.71 \\
\hline Bolivia, La Paz & 14.02 & 15.81 & 10.58 & $0 / 0$ \\
\hline
\end{tabular}

by a fusion of the ancestral $\mathrm{X}_{1}$ and $\mathrm{X}_{2}$ chromosomes. The $\mathrm{X}$ chromosome relative length in Bolivian $P$. rufotuberculatus was significantly similar to the sum of chromosomes $\mathrm{X}_{1}$ and $\mathrm{X}_{2}$ of the individuals from Ecuador and Colombia. Hence, the fusion of the $X_{1}$ and $X_{2}$ chromosomes is the most likely explanation for the emergence of the XY system in P. rufotuberculatus from Bolivia.

Location of $45 \mathrm{~S}$ ribosomal clusters - Another chromosome variation found within $P$. rufotuberculatus was the chromosomal position of the major ribosomal DNA loci. In the Bolivian individuals, the rDNA clusters were located on both sex chromosomes (Fig. 4F-J, bottom row), while in individuals from Colombia and Ecuador they were on one autosomal pair (Fig. 4A-E, top row).

The analysis of 92 triatomine species so far, revealed that the chromosomal position of the $45 \mathrm{~S}$ ribosomal clusters was a species specific character. ${ }^{(21)}$ Intraspecific variation in the rDNA clusters location is an exceptional event within Hemiptera, reported only in one triatomine species: T. infestans. ${ }^{(19,36)}$ Another apparently intraspecific variation reported in Rhodnius ecuadoriensis from Peru and Ecuador by Pita et al. ${ }^{(37)}$ turned out to be explained by the existence of different species. ${ }^{(38)}$

In the Triatomini tribe, the location of rDNA clusters on one autosomal pair is by far the most frequent pattern and is considered as the ancestral character for this group. ${ }^{(17,21)}$ This trend is more acute in the North American lineage, since the autosomal rDNA location is highly conserved including all Panstrongylus species analysed hitherto. ${ }^{(21)}$ Therefore, the sex chromosome location pattern observed in $P$. rufotuberculatus from Bolivia is a very atypical event and should be considered as an apomorphic character. The movement of the ribosomal clusters from autosomes to both sex chromosomes is probably due to transposition events or ectopic recombination, as observed in other triatomine species. ${ }^{(17,19,21)}$ The mobilisation of rDNA loci from autosomes to sex chromosomes alters the genetic recombination of the involved chromosome regions. The differences in genetic recombination rates among autosomes and sex chromosomes are even more extreme in triatomines considering the achiasmatic nature of sex chromosomes. ${ }^{(17,19,21,39)}$ As a result of the reduced recombination, genetic barriers to gene flow could arise rapidly between populations with different sex chromosome systems and location of ribosomal clusters, leading to their divergence and specia- tion. This was suggested for other insect groups such as Coleoptera and Lepidoptera. ${ }^{(40,41)}$ As gene flow is more restricted between sex chromosomes than autosomes, sex linked genes are particularly efficient to produce post-zygotic barriers ${ }^{(42)}$ and therefore rearrangements involving sex chromosomes can be particularly effective as isolation mechanisms.

Most likely, crosses between $P$. rufotuberculatus individuals with different cytotypes could give rise to an adult progeny, which will include individuals with different numbers of $\mathrm{X}$ chromosomes and chromosomes with ribosomal loci. As a consequence of chromosomal pairing and segregation during both meiotic divisions, this hybrid progeny will produce a number of unbalanced gametes, both in the number of $\mathrm{X}$ chromosomes and of chromosomes carrying ribosomal loci, resulting in reproductive disadvantages. This negative effect is exacerbated by the changes in the number of $\mathrm{X}$ chromosomes because they alter the sex determination control and the gene regulation. A similar effect was described in coleopteran species. ${ }^{(40)}$

We propose that the variability in the number of $\mathrm{X}$ chromosomes and rDNA position observed in $P$. rufotuberculatus populations reflects chromosomal rearrangements leading to reproductive incompatibility between both cytotypes. Hence, it is likely that they do not represent intraspecific variability.

Molecular markers - In concordance with previous phylogenetic analyses, ${ }^{(4,5,6,43)}$ our results support the paraphyletic nature of the Panstrongylus genus and their inclusion within North American Triatomini lineage (Figs 5-7). Furthermore, previous reports and the here obtained results also showed that $P$. rufotuberculatus specimens constitute always a separate clade from the remaining Panstrongylus species. Despite the low number of individuals here analysed (38 for the three molecular markers), phylogenetic trees also showed that all individuals morphologically identified as $P$. rufotuberculatus, including those from Bolivia, are closely related. In conclusion, despite its important chromosomal differences, $P$. rufotuberculatus from Bolivia seems to be a sister group to the remaining $P$. rufotuberculatus individuals of other countries.

The average genetic distances (K2-p) of P. rufotuberculatus from Bolivia to others P. rufotuberculatus varies between $10.7 \%$ to $18.7 \%$ and $10.6 \%$ to $15.8 \%$ for 
$c y t b$ and $c o I$ fragments respectively (Table II). The closest individuals to the Bolivian samples are those from La Guajira (North Colombia) with $10.7 \%$ and $10.6 \%$ K2-p distances respectively. The last ones present the regular chromosomal cytotype (Fig. 4). For $c y t b$ gene, this distance was much greater than the cut-off value of $7.5 \%$ proposed by Monteiro et al. ${ }^{(44)}$ to validate distinct triatomine species. For example, within the closely related species of dimidiata complex (T. dimidiata and those later described as T. mopan ${ }^{(45)}$ and T. huehuetenanguensis $\left.{ }^{(46)}\right)$, the $c y t b$ sequences diverged from $8.0 \%$ to $15.5 \%$. (47) Furthermore, Panstrongylus sister species such as $P$. chinai and P. howardi presented a K2-p distance of $7.8 \%{ }^{(48)}$ For $c o I$ sequences, the value of $10.6 \%$ observed between P. rufotuberculatus from Bolivia and La Guajira was also significantly higher than those reported for other closely related triatomine species, as $7.1 \%$ between Mepraia spinolai and M. gajardoi, ${ }^{(24)}$ or $5.3 \%$ between $T$. sordida and the recently described species $T$. rosai ${ }^{\left({ }^{(9)}\right)}$ In summary, the great genetic differentiation observed between the two chromosomal cytotypes of $P$. rufotuberculatus, either by cyt $b$ and $c o I$ fragments, suggests that both groups should be considered as distinct species.

Within $P$. rufotuberculatus, both mitochondrial markers identified four groups: Bolivia, Mexico, North Colombia, and Ecuador plus central Colombia (Table II, Figs 1, 5-7). Unexpectedly, the genetic K2-p distances observed between these four groups are similar to those observed between different species. This would suggest that $P$. rufotuberculatus is most likely a complex of several taxa (Table II). Therefore, it is essential to analyse a greater number of individuals to confirm these high genetic divergences. Unfortunately, chromosomal data from the Mexican group is unavailable at the moment.

Evolutionary origin of $P$. rufotuberculatus from Bolivia - The close relationship obtained in the phylogenetic analyses and the external morphology similarities suggest that $P$. rufotuberculatus from Bolivia is a sister group of the remaining $P$. rufotuberculatus from other countries (Figs 4-6). Variations in the number of $\mathrm{X}$ chromosomes and the rDNA loci chromosomal location of P. rufotuberculatus from Bolivia are most likely explained by at least two different chromosomal rearrangements. Our hypothesis implies an ancestor with a multiple sex system $\left(\mathrm{X}_{1} \mathrm{X}_{2} \mathrm{Y}\right)$ and ribosomal clusters in one autosomal pair, similar to most Panstrongylus species (including P. rufotuberculatus from Colombia and Ecuador). In this ancestor, the first rearrangement that should have occurred was the fusion of the $X_{1}$ and $X_{2}$ chromosomes, resulting in a single fused X chromosome. Subsequently, the complete mobilisation of the ribosomal loci from one autosome pair to the newly fused $\mathrm{X}$ chromosome may have occurred, followed by another transfer (partial) of rDNA clusters from the X chromosome to the Y chromosome. Considering the regular meiotic segregation of sex chromosomes in Bolivian individuals, and the fact that heterozygous individuals for each chromosomal marker were not detected, it is very likely that both rearrangements occurred a long time ago. This could be supported by the significant genetic distances observed among $P$. rufotuberculatus from Bolivia and its sister groups (Table II).
The present paper reports, for the first time, an extensive morphometric and genetic differentiation within Panstrongylus rufotuberculatus. Important chromosomal differences, that would prevent genetic exchange between both chromosomal groups, are here revealed. This evidence is supported by high values of genetic distances and consistent morphometric differences between the groups. Deeper and widely molecular analyses would help to confirm genetic differentiation observed among $P$. rufotuberculatus from different countries.

All the $P$. rufotuberculatus material from Bolivia here analysed, was collected in 2004 and 2006 and belongs to a single locality (La Paz, Muñecas, Camata), both from domiciliary and peridomiciliary environments. However, the geographical distribution of $P$. rufotuberculatus in Bolivia is wider, since it has already been described in other provinces of La Paz Department (Nor Yungas, Sud Yungas, Caranavi, Inquisivi), associated with anthropic structures, particularly intradomiciles. ${ }^{(8,9,10)}$

A detailed morphological examination and other biological aspects of P. rufotuberculatus from Bolivia are now needed to determine external differences that allow its taxonomic recognition as well as for its formal description as a separated species.

\section{ACKNOWLEDGEMENTS}

To Cristina Aznar (AG University, Cayenne, French Guiana) for allowing us to dissect specimens of her collections from French Guiana. The few pictures from Colombia were kindly provided by Harling Caro-Riano (Universidad de Antioquia, Instituto de Biología, Medellín, Colombia). Ecuadorian collection permit ( $\mathrm{N}^{\circ}$ MAE-DNB-CM-2015-0030) and export permit (N71-2018-EXP-CM-FAU-DNB/MA).

\section{AUTHORS' CONTRIBUTION}

SP, AGP, PL, JPD, TC, AV, CG, YP, LC, SP, GBR and FP contributed to field and/or lab works including processing data; SP and FP conceived the experimental plan and coordinated the resources; JPD performed the morphometric analyses; SP and FP wrote the initial manuscript and all co-authors read, edited and commented the paper. FP, SP, YP and LC are members of the "Sistema Nacional de Investigadores (ANII)" and researchers from the "Programa de Desarrollo de las Ciencias Básicas (PEDECIBA) from Uruguay". The authors declare no competing interest.

\section{REFERENCES}

1. Lent H, Wygodzinsky P. Revision of the Triatominae (Hemiptera: Reduviidae), and their significance as vectors of Chagas disease. Bull Am Mus Nat Hist. 1979; 163: 123-520.

2. Costa J, Dale C, Galvão C, Almeida CE, Dujardin JP. Do the new triatomine species pose new challenges or strategies for monitoring Chagas disease? An overview from 1979-2021. Mem Inst Oswaldo Cruz. 2021; 116: e210015.

3. Rojas de Arias A, Monroy C, Guhl F, Sosa-Estani S, Santos WS, Abad-Franch F. Chagas disease control-surveillance in the Americas: the multinational initiatives and the practical impossibility of interrupting vector-borne Trypanosoma cruzi transmission. Mem Inst Oswaldo Cruz. 2021; 116: e210130.

4. Marcilla A, Bargues MD, Abad-Franch F, Panzera F, Carcavallo RU, Noireau F, et al. Nuclear rDNA ITS-2 sequences reveal poly- 
phyly of Panstrongylus species (Hemiptera, Reduviidae, Triatominae), vectors of Trypanosoma cruzi. Infect Genet Evol. 2002; 1(3): 225-35.

5. Justi SA, Russo CAM, Santos-Mallet JR, Obara MT, Galvão C. Molecular phylogeny of Triatomini (Hemiptera: Reduviidae: Triatominae). Parasit Vectors. 2014; 7: 149.

6. Monteiro FA, Weirauch C, Felix M, Lazoski C, Abad-Franch F. Evolution, systematics, and biogeography of the Triatominae, vectors of Chagas disease. Adv Parasitol. 2018; 99: 265-344.

7. Patterson JS, Barbosa SE, Feliciangeli MD. On the genus Panstrongylus Berg 1879: evolution, ecology and epidemiological significance. Acta Trop. 2009; 110(2-3): 187-99.

8. Noireau F, Bosseno MF, Vargas F, Brenière SF. Apparent trend to domesticity observed in Panstrongylus rufotuberculatus Champion, 1899 (Hemiptera, Reduviidae) in Bolivia. Res Rev Parasitol. 1994; 54(4): 263-4.

9. Dujardin JP, Forgues G, Torrez M, Martinez E, Cordoba C, Gianella A. Morphometric of domestic Panstrongylus rufotuberculatus in Bolivia. Ann Trop Med Parasitol. 1998; 92(2): 219-28.

10. Depickère S, Durán P, López R, Chávez T. Presence of intradomicile colonies of the triatomine bug Panstrongylus rufotuberculatus in Muñecas, La Paz, Bolivia. Acta Trop. 2011; 117(2): 97-100.

11. Wolff M, Castillo D. Domiciliation trend of Panstrongylus rufotuberculatus in Colombia. Mem Inst Oswaldo Cruz. 2002; 97(3): 297-300.

12. Grijalva MJ, Villacís AG, Moncayo AL, Ocaña-Mayorga S, Yumiseva CA, Baus EG. Distribution of triatomine species in domestic and peridomestic environments in central coastal Ecuador. PLoS Negl Trop Dis. 2017; 11(10): e0005970.

13. Barnabé C, Grijalva MJ, Santillán-Guayasamín S, Yumiseva CA, Waleckx E, Brenière SF, et al. Genetic data support speciation between Panstrongylus howardi and Panstrongylus chinai, vectors of Chagas disease in Ecuador. Infect Genet Evol. 2020; 78: 104103.

14. Marín E, Santillán R, Cuba C, Jurberg J, Galvão C. Intra-domiciliary capture of Panstrongylus rufotuberculatus (Champion, 1899) (Hemiptera, Reduviidae, Triatominae) in Piura, Peru. Cad Saude Publica. 2007; 23(9): 2235-8

15. Traviezo-Valles LE, Berkefeld D, Aldana E. Infección natural de Panstrongylus rufotuberculatus (Hemiptera: Reduviidae) al sureste del Estado Lara, Venezuela. Bol Mal Salud Amb. 2008; 48(1): 99-101.

16. Hiwat H. Triatominae species of Suriname (Heteroptera: Reduviidae) and their role as vectors of Chagas disease. Mem Inst Oswaldo Cruz. 2014; 109(4): 452-8.

17. Panzera F, Pita S, Lorite P. Chromosome structure and evolution of Triatominae: a review. In: Guarneri AA, Lorenzo MG, editors. Triatominae - the biology of Chagas disease vectors. Entomology in Focus. Vol. 5. Switzerland: Springer; 2021. pp. 65-99.

18. Crossa RP, Hernández M, Caraccio MN, Rose V, Valente SA, da Costa Valente V, et al. Chromosomal evolution trends of the genus Panstrongylus (Hemiptera, Reduviidae), vectors of Chagas disease. Infect Genet Evol. 2002; 2(1): 47-56.

19. Panzera Y, Pita S, Ferreiro MJ, Ferrandis I, Lages C, Pérez R, et al. High dynamics of rDNA cluster location in kissing bug holocentric chromosomes (Triatominae, Heteroptera). Cytogenet Genome Res. 2012; 138(1): 56-67.

20. Panzera F, Pita S, Nattero J, Panzera Y, Galvão C, Chavez T, et al. Cryptic speciation in the Triatoma sordida subcomplex (Hemiptera, Reduviidae) revealed by chromosomal markers. Parasit Vectors. 2015; 8: 495.
21. Pita S, Lorite P, Cuadrado A, Panzera Y, de Oliveira J, Alevi $\mathrm{KCC}$, et al. High chromosomal mobility of rDNA clusters in holocentric chromosomes of Triatominae, vectors of Chagas disease (Hemiptera-Reduviidae). Med Vet Entomol. 2021; https:// doi.org/10.1111/mve.12552.

22. Rohlf FJ. Rotational fit (Procrustes) methods. In: Rohlf F, Bookstein F, editors. Proceedings of the Michigan Morphometrics Workshop. University of Michigan Museums: Ann Arbor; 1990. p. 227-36.

23. Dujardin JP. A template-dependent semilandmarks treatment and its use in medical entomology. Infect Genet Evol. 2019; 70: 197-207.

24. Calleros L, Panzera F, Bargues MD, Monteiro FA, Klisiowicz DR, Zuriaga MA, et al. Systematics of Mepraia (Hemiptera-Reduviidae): cytogenetic and molecular variation. Infect Genet Evol. 2010; 10(2): 221-8.

25. Monteiro FA, Barrett TV, Fitzpatrick S, Cordon-Rosales C, Feliciangeli D, Beard CB. Molecular phylogeography of the Amazonian Chagas disease vectors Rhodnius prolixus and $R$. robustus. Mol Ecol. 2003; 12(4): 997-1006.

26. Simmons MP, Ochoterena H. Gaps as characters in sequence-based phylogenetic analyses. Syst Biol. 2000; 49(2): 369-81.

27. Paradis E, Schliep K. Ape 5.0: an environment for modern phylogenetics and evolutionary analyses in R. Bioinformatics. 2019; 35(3): 526-8.

28. Darriba D, Taboada GL, Doallo R, Posada D. jModelTest 2: more models, new heuristics and parallel computing. Nat Methods. 2012; 9(8): 772 .

29. Guindon S, Dufayard JF, Lefort V, Anisimova M, Hordijk W, Gascuel $\mathrm{O}$. New algorithms and methods to estimate maximum-likelihood phylogenies: assessing the performance of PhyML 3.0. Syst Biol. 2010; 59(3): 307-21.

30. Ronquist F, Teslenko M, van der Mark P, Ayres DL, Darling A, Höhna S, et al. MrBayes 3.2: efficient Bayesian phylogenetic inference and model choice across a large model space. Syst Biol. 2012; 61(3): 539-42.

31. Klingenberg CP, Leamy LJ, Cheverud JM. Integration and modularity of quantitative trait locus effects on geometric shape in the mouse mandible. Genetics. 2004: 166(4); 1909-21.

32. Dujardin JP, Slice DE. Contributions of morphometrics to Medical Entomology. In: Tibayrenc M, editor. Encyclopedia of infectious diseases modern methodologies. Wiley \& Sons, Inc.; 2006. p. 433-45.

33. Ueshima N. Insecta 6. Hemiptera II: Heteroptera. In: John B, editor. Animal Cytogenetics. Vol. 3. Insecta 6 Gebrüder Bornträger, Berlin-Stuttgart; 1979.

34. Papeschi AG. Sex chromosome polymorphism in species of Belostoma (Belostomatidae, Heteroptera). Hereditas. 1996; 124: 269-74.

35. Poggio MG, Provecho YM, Papeschi AG, Bressa MJ. Possible origin of polymorphism for chromosome number in the assassin bug Zelurus femoralis longispinis (Reduviidae: Reduviinae). Biol J Linn Soc. 2013; 110(4): 757-64

36. Panzera F, Ferreiro MJ, Pita S, Calleros L, Pérez R, Basmadjián Y, et al. Evolutionary and dispersal history of Triatoma infestans, main vector of Chagas disease, by chromosomal markers. Infect Genet Evol. 2014; 27: 105-13.

37. Pita S, Panzera F, Ferrandis I, Galvão C, Gómez-Palacio A, Panzera Y. Chromosomal divergence and evolutionary inferences in Rhodniini based on the chromosomal location of ribosomal genes. Mem Inst Oswaldo Cruz. 2013; 108(3): 376-82.

38. Abad-Franch F, Monteiro FA, Pavan MG, Patterson JS, Bargues MD, Zuriaga MA, et al. Under pressure: phenotypic divergence and convergence associated with microhabitat adaptations in Triatominae. Parasit Vectors. 2021; 14(1): 195 
39. Solari AJ. Autosomal synaptonemal complexes and sex chromosomes without axes in Triatoma infestans (Reduviidae, Hemiptera). Chromosoma. 1979; 72: 225-40.

40. Galián J, Proença SJ, Vogler AP. Evolutionary dynamics of autosomal-heterosomal rearrangements in a multiple-X chromosome system of tiger beetles (Cicindelidae). BMC Evol Biol. 2007; 7: 158.

41. Šíchová J, Nguyen P, Dalíková M, Marec F. Chromosomal evolution in tortricid moths: conserved karyotypes with diverged features. PLoS One. 2013; 8(5): e64520.

42. Sætre GP, Borge T, Lindroos K, Haavie J, Sheldon BC, Primmer C, et al. Sex chromosome evolution and speciation in Ficedula flycatchers. Proc R Soc London B. 2003; 270(1510): 53-9.

43. Kieran TJ, Gordon ERL, Zaldívar-Riverón A, Ibarra-Cerdeña C, Glenn TC, Weirauch C. Ultraconserved elements reconstruct the evolution of Chagas disease-vectoring kissing bugs (Reduviidae: Triatominae). Syst Entomol. 2021; 46: 725-40.

44. Monteiro FA, Donnelly MJ, Beard CB, Costa J. Nested clade and phylogeographic analyses of the Chagas disease vector Triatoma brasiliensis in Northeast Brazil. Mol Phyl Evol. 2004; 32(1): 46-56.
45. Dorn PL, Justi SA, Dale C, Stevens L, Galvão C, Lima-Cordón R, et al. Description of Triatoma mopan sp. $\mathrm{n}$. from a cave in Belize (Hemiptera, Reduviidae, Triatominae). ZooKeys. 2018; 775: 69-95.

46. Lima-Cordón RA, Monroy MC, Stevens L, Rodas A, Rodas GA, Dorn PL, et al. Description of Triatoma huehuetenanguensis sp. n., a potential Chagas disease vector (Hemiptera, Reduviidae, Triatominae). ZooKeys. 2019; 820: 51-70.

47. Monteiro FA, Peretolchina T, Lazoski C, Harris K, Dotson EM, Abad-Franch F, et al. Phylogeographic pattern and extensive mitochondrial DNA divergence disclose a species complex within the Chagas disease vector Triatoma dimidiata. PLoS One. 2013; 8(8): e70974.

48. Villacís AG, Dujardin JP, Panzera F, Yumiseva CA, Pita S, Santillán-Guayasamín S, et al. Chagas vectors Panstrongylus chinai (Del Ponte, 1929) and Panstrongylus howardi (Neiva, 1911): chromatic forms or true species? Parasit Vectors. 2020; 13(1): 226.

49. Alevi KCC, de Oliveira J, Garcia ACC, Cristal DC, Delgado LMG, Bittinelli IF, et al. Triatoma rosai sp. nov. (Hemiptera, Triatominae): a new species of Argentinian Chagas disease vector described based on integrative taxonomy. Insects. 2020; 11(12): 830. 